\title{
ON THE MAXIMUM PRINCIPLE FOR ELLIPTIC OPERATORS
}

\author{
L. Caso, P. Cavaliere And M. Transirico
}

Abstract. In this paper we obtain some estimates for solutions of second order elliptic equations whose leading coefficients are functions of vanishing mean oscillation.

Mathematics subject classification (2000): 35J25, 35B50.

Key words and phrases: Elliptic equations, a priori bounds, discontinuous coefficients.

\section{REFERENCES}

11 A. D. AleKSANDROV, Majorization of solutions of second-order linear equations, Vestnik Leningrad. Univ. 21 no. 1 (1966), 5-25 [English Translation in Amer. Math. Soc. Transl. 68 (1968), 120-143].

[2] H. BERESTYCKI, L. NiRENBERG, S. R. S. VARADHAN, The principal eigenvalue and maximum principle for second-order elliptic operators in general domains, Comm. Pure Appl. Math. 47 (1994), 47-92.

[3] X. CABRÉ, On the Alexandroff - Bakelman - Pucci estimate and the reversed Hölder inequality for solutions of elliptic and parabolic equations, Comm. Pure Appl. Math. 48 (1995), 539-570.

[4] F. ChIARENZA, M. FrasCA, P. LONGO, Interior $W^{2, p}$ estimates for non divergence elliptic equations with discontinuous coefficients, Ricerche Mat. 40 (1991), 149-168.

5] D. Gilbarg, N. S. Trudinger, Elliptic Partial Differential Equations of Second Order, 2nd Edition, Springer, Berlin-Heidelberg (1983).

[6] D. GRECO, Nuove formole integrali di maggiorazione per le soluzioni di un'equazione lineare di tipo ellittico ed applicazioni alla teoria del potenziale, Ricerche Mat. 5 (1956), 126-149.

[7] A. I. KosheleV, On boundedness in $L^{p}$ of solutions of elliptic differential equations, Mat. Sbornik 38 (1956), 359-372.

[8] C. Miranda, Sulle equazioni ellittiche del secondo ordine a coefficienti discontinui, Ann. Mat. Pura Appl. (4) 63 (1963), 353-386.

[9] C. PuCCI, Operatori ellittici estremanti, Ann. Mat. Pura Appl. (4) 71 (1966), 141-170.

[10] C. PuccI, Limitazioni per soluzioni di equazioni ellittiche, Ann. Mat. Pura Appl. (4) 74 (1966), $15-30$.

[11] E. M. SteIn, Harmonic Analysis: Real Variable Methods, Orthogonality, and Oscillatory Integrals, Princeton University Press, Princeton (1993).

[12] M. TRANSIRICO, M. Troisi, Equazioni ellittiche del secondo ordine di tipo non variazionale in aperti non limitati, Ann. Mat. Pura Appl. (4) 152 (1988), 209-226.

[13] M. Transirico, M. Troisi, A. Vitolo, Spaces of Morrey type and elliptic equations in divergence form on unbounded domains, Boll. Un. Mat. Ital. (7) 9B (1995), 153-174.

[14] M. Transirico, M. Troisi, A. Vitolo, BMO spaces on domains of $\mathbb{R}^{n}$, Ricerche Mat. 45 (1996), 355-378.

[15] C. VitAnZA, A new contribution to the $W^{2, p}$-regularity for a class of elliptic second order equations with discontinuous coefficients, Matematiche (Catania) 48 (1993), 287-296.

[16] A. ViTolo, Uniqueness estimates for elliptic equations with discontinuous coefficients on unbounded domains, manuscript.

[17] W. P. ZIEMER, Weakly Differentiable Functions, Springer, Berlin (1989). 